\title{
MS08-03 | Structural Analysis of Chloroplast Tail-Anchored Membrane Protein ReCOGNITION BY ARSA1
}

Hsiao, Chwan-Deng (Academia Sinica, Taipei, TWN)

Tail-anchored (TA) membrane proteins destined for the posttranslational pathway are safely delivered to the endoplasmic reticulum (ER) membrane by a well-known targeting factor, TRC40/Get3, in mammals and yeast. In contrast, the underlying mechanism for translocation of TA proteins in plants remains obscure. How this unique eukaryotic membrane-trafficking system correctly distinguishes different subsets of TA proteins destined for various organelles, including mitochondria, chloroplasts and the ER, is a key long-standing question. Here, we present crystal structures of algae ArsA1 (the Get3 homolog) in a new distinct open state of nucleotide-free and AMPPNP-bound. This 80-kDa protein possesses a monomeric architecture, with two ATPase domains in a single polypeptide chain. It is capable of binding chloroplast (TOC34 and TOC159) and mitochondrial (TOM7) TA proteins based on features of its transmembrane domain as well as the regions right before and after the transmembrane domain. Several helices located above the TA-binding groove comprise the interlocking hook-like motif implicated in TA substrate recognition by mutational analyses. Our data provide new insights into the molecular basis of the highly specific selectivity of algae ArsA1 interactions with the correct sets of TA substrates before membrane targeting in plant cells. 